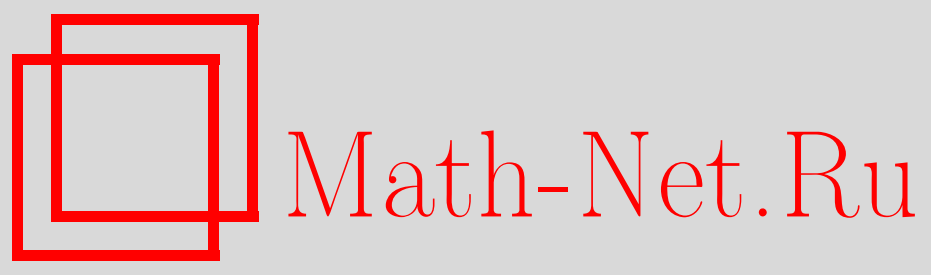

И. А. Пятышев, Пример ограниченного аппроксимативно компактного множества, не являющегося локально компактным, УМН, 2007, том 62, выпуск 5, 163-164

DOI: https://doi.org/10.4213/rm7153

Использование Общероссийского математического портала Math-Net.Ru подразумевает, что вы прочитали и согласны с пользовательским соглашением http://www . mathnet.ru/rus/agreement

Параметры загрузки:

IP: 3.89 .185 .249

26 апреля 2023 г., 07:45:09

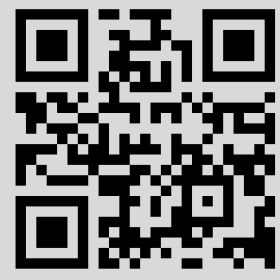




\section{Пример ограниченного аппроксимативно компактного множества, не являющегося локально компактным}

\section{И. А. Пятышев}

Пусть $M$ - некоторое подмножество банахова пространства $X$. Последовательность $\left\{z_{n}\right\}_{n=1}^{\infty} \subset M$ называется минимизирующей для элемента $x \in X$, если $\left\|z_{n}-x\right\| \rightarrow$ $\rho(x, M):=\inf \{\|x-y\|: y \in M\}$ при $n \rightarrow \infty$.

ОПРеДЕлЕниЕ 1. Множество $M$ аппроксимативно компактно, если для любого $x \in X$ всякая минимизирующая последовательность содержит подпоследовательность, сходящуюся к элементу из $M$.

Понятие аппроксимативно компактного множества было введено Н. В. Ефимовым и С. Б. Стечкиным [1] и играет важную роль в теории приближений в нормированных пространствах [2]. Свойства аппроксимативно компактных множеств исследовались многими авторами: см., например, работы [3]-[6].

Всякое ограниченно компактное множество (т. е. множество, пересечение которого с любым замкнутым шаром компактно) является аппроксимативно компактным.

В работе [5] доказано, что в любом бесконечномерном сепарабельном банаховом пространстве существует ограниченное аппроксимативно компактное, но не компактное множество.

ОПределЕние 2. Подмножество $M$ банахова пространства $X$ называется локально компактным, если любая точка этого множества имеет некоторую замкнутую окрестность, пересечение которой с $M$ компактно.

Цель настоящей заметки - показать, что свойства аппроксимативной компактности и локальной компактности не следуют друг из друга.

В произвольном банаховом пространстве несложно построить пример локально компактного, но не аппроксимативно компактного множества. Для этого на единичной сфере достаточно взять счетную последовательность таких элементов $x_{n}$, что расстояние между любыми двумя из них больше $1 / 2$ (см., например, [7; гл. 4, §6]). Множество $M=\left\{x_{n}\right\}$ локально компактно, но не аппроксимативно компактно: для элемента 0 последовательность $\left\{x_{n}\right\}$ является минимизирующей в $M$, но из нее нельзя выбрать сходящуюся подпоследовательность.

Теорема. В любом бесконечномерном сепарабельном банаховом пространстве существует ограниченное аппроксимативно компактное, но не локально компактное множество.

ДокАЗАТЕЛЬСтво существенно использует конструкцию из работы [5]. Отметим, что построенное в [5] аппроксимативно компактное множество является локально компактным.

Пусть $\left\{s_{n}\right\}$ - счетное множество, всюду плотное на единичной сфере $S$ бесконечномерного сепарабельного банахова пространства $X$. Пусть $\Gamma_{n}$ - замкнутая гиперплоскость, опорная к сфере $S$ в точке $s_{n}$, а $\Gamma_{n}^{\prime}=\left\{x \in X: x+s_{n} \in \Gamma_{n}\right\}$ - соответствующее гиперподпространство. Для каждого $n$ в подпространстве $\Gamma_{1}^{\prime} \cap \Gamma_{2}^{\prime} \cap \cdots \cap \Gamma_{n}^{\prime}$ возьмем какой-нибудь вектор $x_{n}$ с $\left\|x_{n}\right\|=1$.

Последовательность $\left\{x_{n}\right\}$ обладает следующим свойством: для любого $x \in X$ и любой числовой последовательности $\left\{t_{n}\right\}$ имеет место неравенство

$$
\liminf _{n \rightarrow \infty}\left\|x-t_{n} x_{n}\right\| \geqslant\|x\| .
$$

Работа выполнена при поддержке РФФИ (грант № 05-01-00962). 
Действительно, если $x=s_{k}$, то при $n \geqslant k$ имеем $s_{k}-t_{n} x_{n} \in \Gamma_{k}$, откуда $\left\|s_{k}-t_{n} x_{n}\right\| \geqslant$ $1=\left\|s_{k}\right\|$, неравенство (1) верно. Следовательно, это неравенство верно и для $x=t s_{k}$ при любых $t>0$ и $k$. Поскольку элементы вида $t s_{k}$ всюду плотны в пространстве $X$, неравенство (1) имеет место для любого $x \in X$.

Положим $y_{n}=s_{n} / n, n=1,2, \ldots$.

Докажем, что множество $M=\bigcup_{n=1}^{\infty}\left(\left[0, x_{n}\right] \cup\left\{y_{n}\right\}\right)$ - искомое (здесь $[a, b]=\{t a+$ $(1-t) b: 0 \leqslant t \leqslant 1\})$.

Очевидно, что множество $M$ ограничено. Оно не локально компактно: для любого $r>0$ замкнутая $r$-окрестность точки $0 \in M$ содержит последовательность $r x_{n}$, из которой нельзя извлечь сходящуюся подпоследовательность. Действительно, если элемент $a$ является предельной точкой для этой последовательности, то $\|a\|=r$ и неравенство (1) для него не выполняется.

Покажем, что множество $M$ аппроксимативно компактно. Пусть $x \in X$ и некоторая последовательность $\left\{z_{n}\right\} \subset M$ является минимизирующей для $x$. Если $x=0$, то $z_{n} \rightarrow 0$. Если же $x \neq 0$, то для некоторого $y_{k}$ имеем $\left\|x-y_{k}\right\|<\|x\|$, а значит, $\rho(x, M)<\|x\|$. Но тогда в силу неравенства (1) и того, что $y_{n} \rightarrow 0$, последовательность $z_{n}$ пробегает по конечному набору точек $y_{n}$ и отрезков $\left[0 ; x_{n}\right]$, а значит, из нее можно извлечь сходящуюся подпоследовательность.

Теорема доказана.

Остается неясным, существует ли аппроксимативно компактное, но не локально компактное множество в произвольном бесконечномерном банаховом пространстве. Неизвестен ответ и на более специальный вопрос: во всяком ли банаховом пространстве существует (выпуклое) аппроксимативно компактное тело? В работе [6] такое тело построено в произвольном рефлексивном пространстве, а в работе [8] - в пространстве $c_{0}$ сходящихся к нулю последовательностей.

\section{Список литературы}

[1] Н. В. Ефимов, С. Б. Стечкин, Докл. АН СССР, 140:3 (1961), 522-524. [2] В. С. Балаганский, Л. П. Власов, УМH, 51:6 (1996), 125-188. [3] I. Singer, Rev. Roumaine Math. Pures Appl., 9:2 (1964), 167-177. [4] С. В. Конягин, И. Г. Царьков, Вестн. Моск. ун-та. Сер. 1. Матем., мех., 1986, № 5, 20-27. [5] П. А. Бородин, УМН, 49:4 (1994), 157-158. [6] П. А. Бородин, Вестн. Моск. ун-та. Сер. 1. Матем., мех., 1999, № 4, 19-21. [7] А. Н. Колмогоров, С. В. Фомин, Элементы теории функций и функционального анализа, Наука, М., 1989. [8] И. А. Пятышев, Вестн. Моск. ун-та. Сер. 1. Матем., мех., 2005, №3, 57-59.

\section{И. А. Пятышев (I. A. Pyatyshev)}

Московский государственный университет им. М.В̈. Ломоносова
Представлено В. М. Тихомировым

Принято редколлегией 08.06.2007 